\title{
Differentiating Majorana from Andreev bound states in a superconducting circuit
}

\author{
Konstantin Yavilberg, ${ }^{1}$ Eran Ginossar, ${ }^{2}$ and Eytan Grosfeld ${ }^{1}$ \\ ${ }^{1}$ Department of Physics, Ben-Gurion University of the Negev, Be'er-Sheva 84105, Israel \\ ${ }^{2}$ Advanced Technology Institute and Department of Physics, University of Surrey, Guildford GU2 7XH, United Kingdom
}

(Received 8 August 2019; revised manuscript received 10 December 2019; published 27 December 2019)

\begin{abstract}
We investigate the low-energy theory of a one-dimensional finite capacitance topological Josephson junction. Charge fluctuations across the junction couple to resonant microwave fields and can be used to probe microscopic excitations such as Majorana and Andreev bound states. This marriage between localized microscopic degrees of freedom and macroscopic dynamics of the superconducting phase, leads to unique spectroscopic patterns which allow us to reveal the presence of Majorana fermions among the low-lying excitations.
\end{abstract}

DOI: $10.1103 /$ PhysRevB.100.241408

Introduction. Quantized supercurrent oscillations in Josephson junctions strongly coupled to cavity photons, within the framework of circuit quantum electrodynamics (cQED), have become a prominent source, not only for the development of quantum processors based on the transmon [1-8], but also in the study of mesoscopic solid-state phenomena. Their high- $Q$ superconducting resonator environment and the nonlinearity of the junction, allow precise control and high resolution microwave probing while maintaining strong coherence throughout the system. New generation hybrid devices combine additional solid-state components [9-18] in order to enhance their tunability, control their responsiveness to external fields, and develop a framework that can support unique quantum states, which may be difficult to probe and control in other systems.

A promising direction is to include solid-state materials that when embedded inside a Josepshon junction can realize topological superconductivity via the proximity effect. Prime candidates include one-dimensional realizations of a helical liquid, including nanoribbons made of topological insulators such as $\mathrm{Bi}_{2} \mathrm{Se}_{3}$ and $\mathrm{Bi}_{2} \mathrm{Te}_{3}$ [19-25], or strong spin-orbit semiconductors such as InAs [26-30]. The resulting topological Josephson junctions can nucleate Majorana modes whose properties can be harnessed to generate improved qubit devices [31-38]. So far mostly pristine topological cQED devices were theoretically studied. However, in present experimental realizations a combination of Majorana and Andreev bound states is expected to be present within the junction's weak link [39-48]. While their hybrid properties should play a crucial role in the development of current and future qubit devices, viable experimental methods to differentiate between their signatures in CQED are still wanting.

In this Rapid Communication we develop a methodology which allows one to study a floating mesoscopic topological Josephson junction, and to predict the experimental signatures of its low-energy excitations. The theoretical challenge stems from the interplay of the microscopic (bound states) and macroscopic (transmon) degrees of freedom controlling the dynamics of the junction. Our method identifies the relevant low-energy degrees of freedom and derives their combined dynamics. Using this method, we extract the dipole transitions of the device, which reveal the presence of bound states in the junction through a fine structure around the plasma frequency. These transitions target processes related to the Andreev bound states and their interaction with the Majorana fermions, and contain revealing signatures of these two types of bound states.

Description of the model. We consider a one-dimensional helical liquid bridging two superconducting islands, giving rise to Majorana and Andreev bound states. For concreteness we model a topological insulator nanowire with an applied magnetic flux $\Phi$ [23-25]; we should note that our results also apply to other realizations with small modifications, such as semiconductor nanowires $[27,28]$. The nanowire is connected in parallel to a regular Josephson junction with strong Josephson coupling (see Fig. 1). The anharmonic spectrum of the transmon, which is required for a viable qubit device, can be controlled by a side gate. Due to quantum confinement in the radial direction of the nanowire, multiple bands exist separated by $\sim v / R$, with $R$ the nanowire's radius and $v$ the Fermi velocity. We tune the magnetic flux close to $\Phi=\frac{h c}{2 e}$, noting that any discrepancy from this value will open a finite magnetic gap $\Delta_{B}$ in the Dirac spectrum [49], and set the chemical potential to $|\mu|<\frac{v}{R}-\Delta_{B}$. This ensures that only the lowest nondegenerate band is occupied, thus creating effectively a one-dimensional system. For convenience and without affecting the main results, we have set $\mu=0$ throughout.

For a complete model of the system we consider the action $S=S_{\mathrm{sc}}+S_{W}+S_{\text {tun }}+S_{J}$. The first term $S_{\mathrm{sc}}=$ $\sum_{j} \int d t d z \Psi_{j}^{\dagger} G_{j}^{-1} \Psi_{j}$, describes the proximity-induced superconductivity in the left $(j=1)$ and right $(j=2)$ islands, given by the Green's function $G_{j}^{-1}=i \partial_{t}-\left(i v \partial_{z} \sigma_{y}+\right.$ $\left.\Delta_{B} \sigma_{z}+\frac{\partial_{t} \phi_{j}}{2}\right) \tau_{z}+\Delta \sigma_{y} \tau_{y}$. Here $\sigma_{i}$ and $\tau_{i}$ are Pauli matrices in spin and Nambu space, respectively, with the spinor $\Psi=$ $\frac{1}{\sqrt{2}}\left(\psi_{\uparrow}, \psi_{\downarrow}, \psi_{\uparrow}^{\dagger}, \psi_{\downarrow}^{\dagger}\right)^{T}$. The superconducting phase in the pairing term $\Delta e^{i \phi_{j}(t)}$ is treated beyond the mean-field approximation, which allows us to take into account the effects of charge fluctuations. In writing $S_{\text {sc }}$ we employed the gauge transformation $\Psi \rightarrow e^{[i \phi(t) / 2] \tau_{z}} \Psi$ which removes the phase from the pairing term and adds a coupling of $\partial_{t} \phi_{j}(t)$ to 


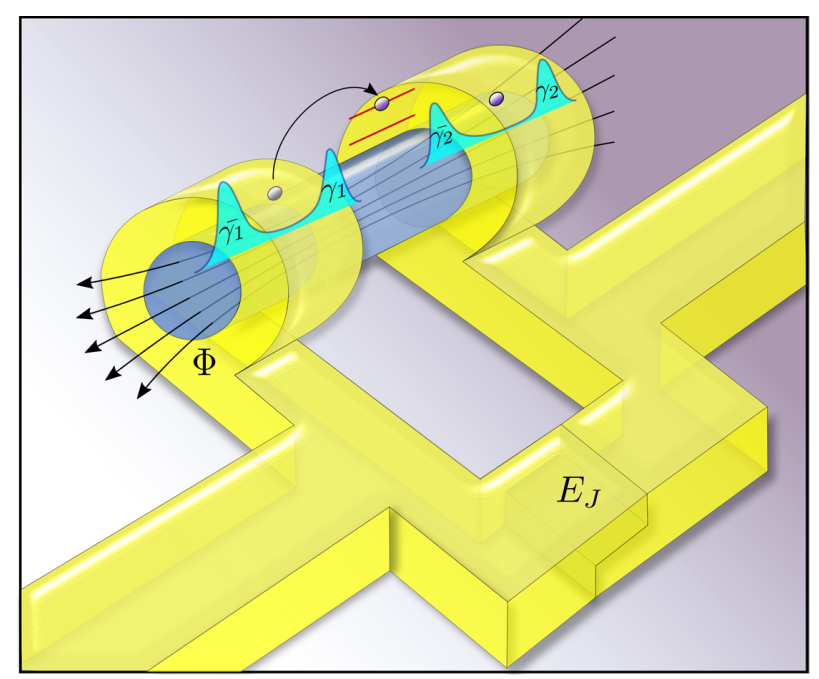

FIG. 1. Schematic description of the model. A helical liquid bridging two superconducting islands nucleates Majorana and Andreev bound states. The states mediate charge transfer between the islands through single electron processes. A parallel Josephson junction generates tunneling of Cooper pairs of strength $E_{J}$. The helical liquid is depicted as a topological insulator nanowire threaded with a constant magnetic flux $\Phi$.

the density $\Psi_{j}^{\dagger} \Psi_{j}$. The weak link is modeled as a twostate system, given by $S_{W}=\int d t \mathcal{C}^{\dagger}\left(i \partial_{t}-\varepsilon \tau_{z}-\Delta_{B} \sigma_{z} \tau_{z}\right) \mathcal{C}-$ $U c_{\uparrow}^{\dagger} c_{\uparrow} c_{\downarrow}^{\dagger} c_{\downarrow}$, where $\mathcal{C}=\frac{1}{\sqrt{2}}\left(c_{\uparrow}, c_{\downarrow},-c_{\uparrow}^{\dagger},-c_{\downarrow}^{\dagger}\right)^{T}$ with the operators $c_{\uparrow}, c_{\downarrow}$ representing low-energy modes with spin orientation along the nanowire. This is justified due to the finite size of the weak link and the resulting level quantization. We have also included $\varepsilon$ which can be controlled by a local gate operating on the weak link, and a repulsive Coulomb interaction $U$. We assume that the coupling of the weak link to the islands is given by a tunneling term of the form $S_{\text {tun }}=\sum_{j}\left(\lambda \int d t \Psi_{j}^{\dagger}(0) e^{\left[i \phi_{j}(t) / 2\right] \tau_{z}} \mathcal{C}+\right.$ H.c. $)$. This term can be realized by locally narrowing the nanowire near the edges of the weak link [37], which opens a magnetic gap and results in tunnel barriers. An alternative approach which does not require a tunnel junction is presented in [49]. The action of the parallel regular Josephson junction is given by $S_{J}=$ $\int d t\left[\frac{\left(\dot{\phi}_{1}-\dot{\phi}_{2}\right)^{2}}{16 E_{C}}+\frac{\left(\dot{\phi}_{1}+\dot{\phi}_{2}\right)^{2}}{16 E_{C}^{\prime}}+E_{J} \cos \left(\phi_{1}-\phi_{2}\right)\right]$. Here $E_{C}$ and $E_{C}^{\prime}$ define the scale of the charging effect, originating from the finite capacitance of the mesoscopic device, with the ratio $E_{C}^{\prime} / E_{C} \leqslant 1$ controlling the strength of the mutual capacitance. Throughout we will assume that the system operates in the transmon regime $E_{C}, E_{C}^{\prime} \ll E_{J}[1]$, where $E_{J}$ is the Josephson energy. We consider the case where there is no flux penetration through the loop created by the two parallel junctions (see Fig. 1).

The dynamics of the mesoscopic topological junction is dominated by a set of degrees of freedom for which we now derive an effective theory. The theory accounts for the interaction of Cooper pairs with the bound states, by systematically integrating all highly fluctuating degrees of freedom [49]. This results in an effective Hamiltonian $H_{\text {eff }}=H_{\mathcal{C}}+H_{\gamma}+H_{T}$ which we later use for our main analysis of the system. Here
$H_{T}$ is a modified transmon Hamiltonian

$$
H_{T}=4 E_{C}\left(\hat{n}-n_{g}\right)^{2}+E_{C}^{\prime}\left(\hat{N}^{2}+2 \alpha \mathcal{C}^{\dagger} \tau_{z} \mathcal{C} \hat{N}\right)-E_{J} \cos (\hat{\varphi}),
$$

where $\hat{n}=\frac{1}{2}\left(\hat{n}_{1}-\hat{n}_{2}\right)$ is the relative number of Cooper pairs between the islands, $\hat{N}=\hat{n}_{1}+\hat{n}_{2}$ is the total number of Cooper pairs exceeding neutrality in the islands, and $\hat{\varphi}=$ $\hat{\phi}_{1}-\hat{\phi}_{2}$ is the phase difference conjugate to $\hat{n}$. The operator $e^{-i q \hat{\varphi}}\left(e^{i q \hat{\varphi}}\right)$ transfers a charge $q$ from the left to the right (right to the left) island. We redefined $E_{C}$ and $E_{C}^{\prime}$ to include the capacitance of both the topological Josephson junction and the rest of the transmon. A side gate generates an offset charge $n_{g}$, measured in units of the Cooper pair charge. The parameter $\alpha \equiv \alpha_{c}+\frac{\lambda^{2}}{v \Delta}\left(1-\frac{\Delta_{B}^{2}}{\Delta^{2}}\right)^{-1}$, controls the electrostatic interaction between the weak link and the islands, and is comprised of two contributions: one is capacitive, given by a phenomenological parameter $\alpha_{c}$ which depends on the geometry of the device, and the other is a consequence of the induced superconductivity in the weak link.

The weak link is governed by

$$
\begin{aligned}
H_{\mathcal{C}}= & \left(\tilde{\varepsilon}+\Delta_{B}\right) c_{\uparrow}^{\dagger} c_{\uparrow}+\left(\tilde{\varepsilon}-\Delta_{B}\right) c_{\downarrow}^{\dagger} c_{\downarrow}+\tilde{U} c_{\uparrow}^{\dagger} c_{\uparrow} c_{\downarrow}^{\dagger} c_{\downarrow} \\
& +2 \Gamma \cos (\hat{\varphi} / 2)\left(e^{i \hat{\delta}} c_{\uparrow} c_{\downarrow}+e^{-i \hat{\delta}} c_{\downarrow}^{\dagger} c_{\uparrow}^{\dagger}\right),
\end{aligned}
$$

where $\Gamma=\lambda^{2} / v$ is the induced pairing and $\hat{\delta}=\frac{1}{2}\left(\hat{\phi}_{1}+\hat{\phi}_{2}\right)$ is the average phase conjugate to $\hat{N}$. The operator $e^{-i q \hat{\delta}}$ transfers a charge $q$ from the weak link to the islands. The induced pairing has emerged from the integration of high-energy degrees of freedom so it should satisfy $\Gamma \ll \Delta$. To ensure the presence of a low-lying Andreev bound state we further assume $\Gamma \ll E_{J}$. The rest of the parameters were modified to $\tilde{U}=U+2 E_{C}^{\prime} \alpha^{2}$ and $\tilde{\varepsilon}=\varepsilon+E_{C}^{\prime} \alpha^{2}$. The coupling to the Majorana fermions is given by

$$
H_{\gamma}=w e^{i \hat{\delta} / 2}\left[i e^{i \hat{\varphi} / 4}\left(c_{\uparrow}+c_{\downarrow}\right) \gamma_{1}+e^{-i \hat{\varphi} / 4}\left(c_{\uparrow}-c_{\downarrow}\right) \bar{\gamma}_{2}\right]+\text { H.c. },
$$

where $\gamma_{1}, \bar{\gamma}_{2}$ are Hermitian operators denoting the Majorana fermions localized near the weak link and $w \sim \sqrt{\Gamma \Delta_{B}}$. We assume negligible hybridization with the Majorana fermions $\bar{\gamma}_{1}, \gamma_{2}$ at the nanowire's remote ends and exclude them from the model [49]. As the parity in each island is given by the occupation of the nonlocal zero modes $f_{1}$ and $f_{2}$, defined by $\gamma_{1}=i\left(f_{1}^{\dagger}-f_{1}\right)$ and $\bar{\gamma}_{2}=f_{2}^{\dagger}+f_{2}[50]$, the transfer of charge in Eq. (3) is also accompanied by a change of fermionic parity. Since the system is only capacitively shunted the total number of particles is conserved and can be fixed by a neutrality condition $2 \hat{N}+\hat{n}_{W}=0$, where $\hat{n}_{W}=c_{\uparrow}^{\dagger} c_{\uparrow}+c_{\downarrow}^{\dagger} c_{\downarrow}$. With this constraint and the different parity combinations, we end up with eight different subspaces denoted by $\left|p_{1}, p_{2}, \sigma_{w}\right\rangle$, where $p_{j}=0,1$ indicates the occupation of $f_{j}$ and $\sigma_{w}=0, \uparrow, \downarrow, \uparrow \downarrow$ correspond to the spin configurations in the weak link.

Spectroscopic signatures of bound states. To study the effect of the bound states on the spectroscopic signatures, we take for a long wire $U \sim E_{C}$ and $\alpha_{c} \ll 1$. We first consider the case where the Majorana fermions are absent, by setting $\Delta_{B}=$ 0 . The resulting Hamiltonian conserves fermionic parity. By projecting $H_{\text {eff }}$ onto the $\{|0,0,0\rangle,|0,0, \uparrow \downarrow\rangle\}$ subspace we 

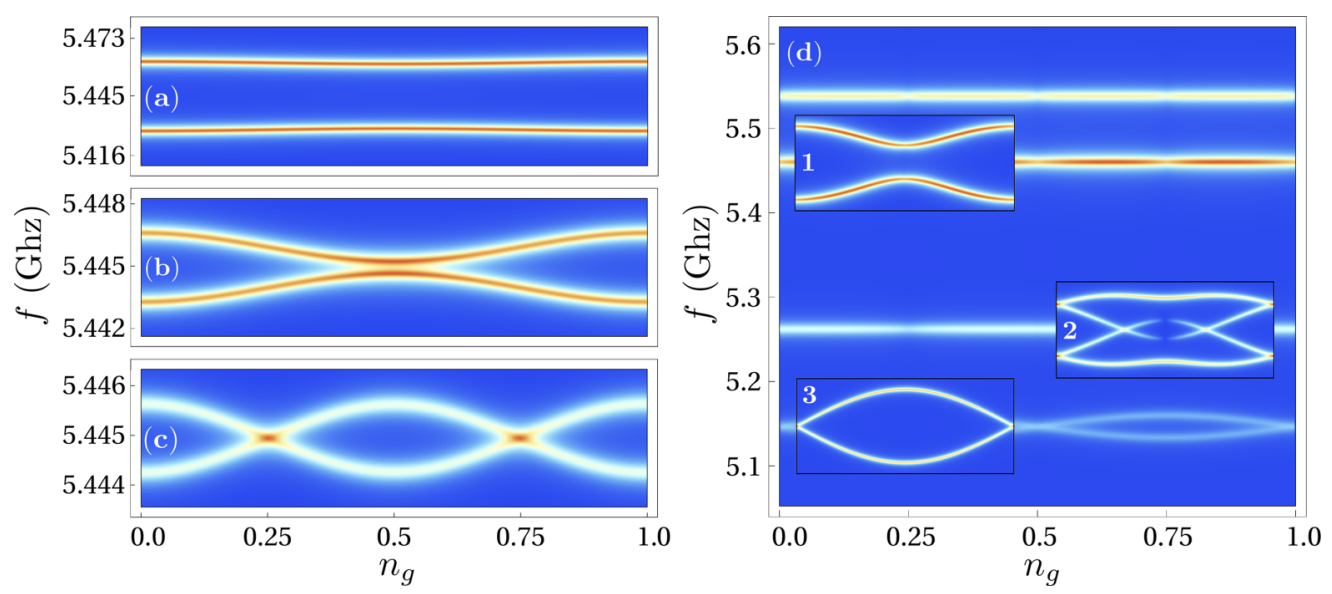

FIG. 2. Predicted spectroscopic signatures for different configurations of bound states. Dipole transition lines are presented as a function of $n_{g}$ for the lowest-energy sector. We use values typical for a transmon, taking $E_{C} / 2 \pi=0.4 \mathrm{GHz}, E_{J} / E_{C}=27, \Delta / E_{J}=10$, and $\varepsilon / E_{C}=0.8$ with $U / E_{C}=0.6$ for the weak-link parameters. We have also set $E_{C}^{\prime} / E_{C}=1$, as any deviation of this ratio from unity results in a simple renormalization of the rest of the parameters. In the case $\Delta_{B}=0$ we look at three different coupling strengths: (a) $\Gamma / E_{C}=0.5, \kappa / 2 \pi=3 \mathrm{MHz}$, (b) $\Gamma / E_{C}=0.12, \kappa / 2 \pi=0.4 \mathrm{MHz}$, and (c) $\Gamma / E_{C}=0.04, \kappa / 2 \pi=0.3 \mathrm{MHz}$. All lines have the same periodicity $n_{g} \rightarrow n_{g}+1$, indicating tunneling of solely Cooper pairs. In (d) the magnetic gap is increased to $\Delta_{B} / E_{C}=11.8$, which results in additional transition lines. With the presence of Majorana fermions all transition lines exhibit an $n_{g} \rightarrow n_{g}+1 / 2$ periodicity due to single particle tunneling. We have set $\Gamma / E_{C}=0.01, \varepsilon / E_{C}=1.3$, and $U / E_{C}=2.3$. Patterns fall into three types according to their behavior at $n_{g}=1 / 4$, as seen in the magnified insets. Insets 1 and 3 show patterns characteristic to Andreev bound states; however, due to the mixing of fermionic parity they develop a gap at $n_{g}=1 / 4$ of size $\Delta f=0.57 \mathrm{MHz}$ and $\Delta f=26.4 \mathrm{MHz}$, respectively. Inset 2 shows the effect of Majorana fermions and Andreev bound states hybridization in its most distinctive form. The central lines show spectral holes, and are enveloped by a form of type 1 with an increased gap $\Delta f=1.86 \mathrm{MHz}$. In insets 1 and 2 we used $\kappa / 2 \pi=0.1 \mathrm{MHz}$, and in inset 3 we used $\kappa / 2 \pi=0.9 \mathrm{MHz}$.

obtain

$$
\mathcal{H}=\left(\begin{array}{cc}
H_{T}\left[n_{W}=0\right] & 2 \Gamma \cos (\hat{\varphi} / 2) \\
2 \Gamma \cos (\hat{\varphi} / 2) & H_{T}\left[n_{W}=2\right]+2 \tilde{\varepsilon}+\tilde{U}
\end{array}\right),
$$

where $n_{W}=\left\langle p_{1}, p_{2}, \sigma_{W}\left|\hat{n}_{W}\right| p_{1}, p_{2}, \sigma_{W}\right\rangle$. We can get a qualitative picture by focusing on solutions with $\varphi \ll 1$, as is characteristic to the transmon regime. Ignoring the effect of the offset charge, a straightforward diagonalization of Eq. (4) gives us two independent sectors $\mathcal{H}_{ \pm} \simeq 4 E_{C} \hat{n}^{2}+E_{J} \hat{\varphi}^{2} / 2 \pm$ $\sqrt{\left(E_{C}^{\prime}+2 \varepsilon+U\right)^{2}+16 \Gamma^{2}} / 2$, corresponding to two shifted harmonic oscillators whose frequency $\omega_{p}=\sqrt{8 E_{C} E_{J}}$ is the plasma frequency. Higher order contributions in $\varphi$ lead to an anharmonicity of order $E_{C}$. The split spectrum is a result of the Andreev bound states inducing additional charge fluctuations in the weak link [51,52] as compared to the traditional transmon. One can appreciate this by looking at the charge distribution given by $\left\langle\hat{n}_{W}\right\rangle_{ \pm} \simeq 1 \pm \tanh \left(\frac{E_{C}^{\prime}+2 \varepsilon+U}{4 \Gamma}\right)$ in each sector of $\mathcal{H}_{ \pm}$. This was calculated using the eigenstates of Eq. (4) with $\varphi \ll 1$. To obtain a quantitative description of the model we construct the Hilbert space using the eigenstates of $\hat{n}$ and $\hat{n}_{W}$. Since only Cooper pairs tunnel in this regime, the $n_{W}=0$ sector imposes $n \in \mathbb{Z}$, while in the $n_{W}=2$ sector $n \in \mathbb{Z}+1 / 2$, due to the absence of a Cooper pair in one of the islands. This division between the sectors is illustrated in the dependence of the energy spectrum on $n_{g}$ (the charge dispersion [1,3]), and can be seen in the spectroscopic signatures (Fig. 2). The charge fluctuations between the two sides of the junction result in a coupling of the system to an electromagnetic field via the dipole moment, proportional to $\hat{n}$. The spectroscopic pattern is given by the cavity response $S_{i j}(\omega)=|\langle i|\hat{n}| j\rangle|^{2}\left(\frac{\kappa^{2}}{\left[\omega-E_{i j}\left(n_{g}\right)\right]^{2}+(\kappa / 2)^{2}}\right)$, calculated as a function of $n_{g}$. Here $\omega$ is the frequency of the photons, $\kappa$ is determined by the quality factor of the cavity, and $E_{i j}\left(n_{g}\right)$ is the energy difference between the $i$ th and $j$ th level of $H_{\text {eff }}$. As in the traditional transmon, the dominant dipole transitions are between neighboring levels separated by $\sim \omega_{p}$. Here, however, each sector of $\mathcal{H}_{ \pm}$contributes a transition line shifted with respect to its partner by $n_{g} \rightarrow n_{g}+1 / 2$, which results in a doubletlike pattern. For $\Gamma \rightarrow 0$ the transition lines cross at the degeneracy points $n_{g}=1 / 4+\mathbb{Z} / 2$ in a manner which is seen in experimental measurements of the transmon [3] and is usually a result of quasiparticle poisoning.

The dependence of the charge distribution $\left\langle\hat{n}_{W}\right\rangle_{ \pm}$on the local gate $\varepsilon$ suggests a special symmetry point at $\varepsilon=-\left(E_{C}^{\prime}+\right.$ $U) / 2$. By tuning the system to this point, each sector of $\mathcal{H}_{ \pm}$contributes a single fermion to the weak link which occupies the Andreev bound state and results in an added pair of transition lines to Figs. 2(a)-2(c). This behavior is superficially similar to the Majorana-transmon [7,12], where neighboring Majorana fermions hybridize in the weak link. By shifting the gate away from this finely tuned symmetry point, one can easily distinguish between the role performed by Andreev bound states and that of neutral Majorana fermions, as the latter are not affected by the gate.

We now change the flux in order to open a magnetic gap $\Delta_{B} \neq 0$. This uncovers the inherent differences between Andreev bound states and Majorana fermions, as observed in their distinct sensitivity to $n_{g}$. The two combined bound states result in a rich array of parity configurations due to single fermion transfer. The $n_{w}=0,2$ subspaces now have variants of even and odd fermionic parity on each side while in total keeping a symmetric combination $\left(p_{1}=p_{2}\right)$. In both variants a spin singlet is transferred between the islands without any 

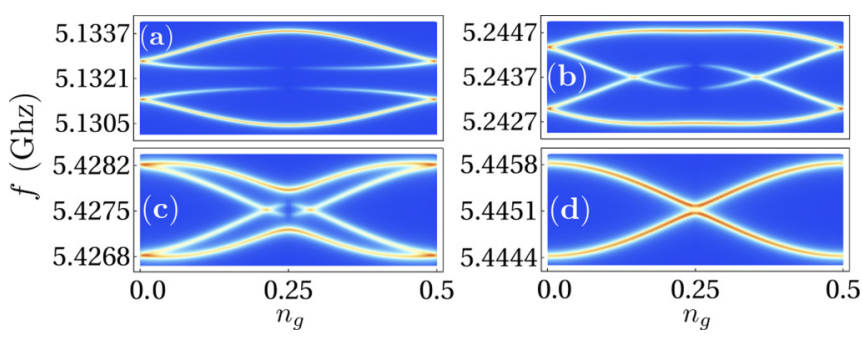

FIG. 3. Dependence of the bound-states hybrid pattern on the magnetic gap. Here we show dipole transitions as a function of $n_{g}$, following the pattern in inset 2 of Fig. 2, for the values (a) $\Delta_{B}=$ $11 E_{C}$, (b) $\Delta_{B}=11.3 E_{C}$, (c) $\Delta_{B}=12 E_{C}$, and (d) $\Delta_{B}=12.5 E_{C}$. The patterns have a similar dipole magnitude and are plotted with $\kappa / 2 \pi=0.1 \mathrm{MHz}$. The rest of the parameters are the same as in Fig. 2(d).

direct response to $\Delta_{B}$. The $n_{W}=1$ subspaces, on the other hand, which have an asymmetric parity combination $\left(p_{1} \neq\right.$ $p_{2}$ ), accommodate spin-polarized Andreev bound states which hybridize with the Majorana fermions and result in a Zeeman splitting around the anharmonic transmon levels. The symmetric and asymmetric subspaces couple to each other with strength $\sim \sqrt{\Gamma \Delta_{B}}$, and due to single fermion tunneling the periodicity of the spectroscopic patterns is halved with respect to $n_{g}$. All the dipole transitions (see Fig. 2) are grouped into bands with a bandwidth determined by the charge dispersion $\sim e^{-\sqrt{8 E_{J} / E_{C}}}$. When approaching $n_{g}=1 / 4$, the correlations between the subspaces cluster into three distinct forms. Two of the forms have transition lines which can be distinguished by their curvature near $n_{g}=1 / 4$ and a shift of $n_{g} \rightarrow n_{g}+1 / 4$, one having an $\sim \cos ^{2}\left(2 \pi n_{g}\right)$ dependence while the other is $\sim \sin ^{2}\left(2 \pi n_{g}\right)$. This shift in the offset charge persists even for very small values of $\Delta_{B}$, and represents the difference in the energy spectrum between the symmetric and asymmetric parity subspaces. The third form, which is characterized by the hybridization between Majorana and Andreev bound states, shows forbidden transitions near $n_{g}=1 / 4$, indicating the presence of Majorana fermions. The exact pattern is not rigid as can be seen in Fig. 3. By increasing $\Delta_{B}$ the band gradually changes its curvature and reduces its gap size, while still retaining the forbidden transitions. Thus a sweep of the magnetic flux reveals the unmistakable transition lines characteristic of Majorana fermions. Note that all three patterns can change from one form to the other, as varying the flux will inevitably create level crossings when $\Delta_{B} \sim \omega_{p}$.

Conclusion. In this work we investigated the physics of coupled low-energy bound states in a one-dimensional topological Josephson junction, where charging effects play an important role, by developing an effective theory for the physics of the weak link. We have shown that we can tune the junction between two remarkably different behaviors. The first is characterized by the absence of Majorana fermions, with Andreev bound states generating dipole transitions similar to those found in the traditional transmon. The second, in contrast, marked by the nucleation of Majorana fermions, displays a striking difference in the vicinity of the $n_{g}=1 / 4$ point, where some of the transition lines develop a vanishing intensity. The reason for this behavior is traced to a destructive interference between different parity states, mediated by the Majorana fermions. This signature emerges despite the presence of Andreev bound states and is distinct from their behavior. While zeros in the intensities at $n_{g}=1 / 4$ might occur accidentally also in the absence of Majorana fermions, the application of a local gate reveals the persistent neutrality of the Majorana fermions by maintaining a sharp zero at this value of the offset charge. Such a measurement would benefit from the unparalleled sensitivity of the cQED framework, which already accomplished experimental feats ranging from the detection of two-level defects in the oxides [53] to singlephoton detection $[54,55]$ in the cavity. The same noninvasive methods can allow unprecedented accuracy for the detection of Majorana and Andreev bound states, as well as the characterization of this hybrid Majorana-Andreev-transmon model, a natural precursor for a qubit device.

Acknowledgments. This project has received funding from the European Union's Horizon 2020 Research and Innovation programme under Grant Agreement No. 766714. K.Y. and E.Gr. acknowledge support from the Israel Science Foundation under Grant No. 1626/16.
[1] J. Koch, T. M. Yu, J. Gambetta, A. A. Houck, D. I. Schuster, J. Majer, A. Blais, M. H. Devoret, S. M. Girvin, and R. J. Schoelkopf, Phys. Rev. A 76, 042319 (2007).

[2] J. Majer, J. M. Chow, J. M. Gambetta, J. Koch, B. R. Johnson, J. A. Schreier, L. Frunzio, D. I. Schuster, A. A. Houck, A. Wallraff, A. Blais, M. H. Devoret, S. M. Girvin, and R. J. Schoelkopf, Nature (London) 449, 443 (2007).

[3] J. A. Schreier, A. A. Houck, J. Koch, D. I. Schuster, B. R. Johnson, J. M. Chow, J. M. Gambetta, J. Majer, L. Frunzio, M. H. Devoret, S. M. Girvin, and R. J. Schoelkopf, Phys. Rev. B 77, 180502(R) (2008).

[4] V. E. Manucharyan, J. Koch, L. I. Glazman, and M. H. Devoret, Science 326, 113 (2009).

[5] F. Hassler, A. R. Akhmerov, and C. W. J. Beenakker, New J. Phys. 13, 095004 (2011).

[6] M. H. Devoret and R. J. Schoelkopf, Science 339, 1169 (2013).
[7] E. Ginossar and E. Grosfeld, Nat. Commun. 5, 4772 (2014).

[8] T. Li, W. A. Coish, M. Hell, K. Flensberg, and M. Leijnse, Phys. Rev. B 98, 205403 (2018).

[9] A. Imamoğlu, Phys. Rev. Lett. 102, 083602 (2009).

[10] Y. Kubo, C. Grezes, A. Dewes, T. Umeda, J. Isoya, H. Sumiya, N. Morishita, H. Abe, S. Onoda, T. Ohshima, V. Jacques, A. Dréau, J.-F. Roch, I. Diniz, A. Auffeves, D. Vion, D. Esteve, and P. Bertet, Phys. Rev. Lett. 107, 220501 (2011).

[11] J.-M. Pirkkalainen, S. U. Cho, J. Li, G. S. Paraoanu, P. J. Hakonen, and M. A. Sillanpää, Nature (London) 494, 211 (2013).

[12] K. Yavilberg, E. Ginossar, and E. Grosfeld, Phys. Rev. B 92, 075143 (2015).

[13] Z.-B. Feng, Phys. Rev. A 91, 032307 (2015).

[14] T. W. Larsen, K. D. Petersson, F. Kuemmeth, T. S. Jespersen, P. Krogstrup, J. Nygård, and C. M. Marcus, Phys. Rev. Lett. 115, 127001 (2015). 
[15] G. de Lange, B. van Heck, A. Bruno, D. J. van Woerkom, A. Geresdi, S. R. Plissard, E. P. A. M. Bakkers, A. R. Akhmerov, and L. DiCarlo, Phys. Rev. Lett. 115, 127002 (2015).

[16] Y. Tabuchi, S. Ishino, A. Noguchi, T. Ishikawa, R. Yamazaki, K. Usami, and Y. Nakamura, Science 349, 405 (2015).

[17] J. G. Kroll, W. Uilhoorn, K. L. van der Enden, D. de Jong, K. Watanabe, T. Taniguchi, S. Goswami, M. C. Cassidy, and L. P. Kouwenhoven, Nat. Commun. 9, 4615 (2018).

[18] J. I.-J. Wang, D. Rodan-Legrain, L. Bretheau, D. L. Campbell, B. Kannan, D. Kim, M. Kjaergaard, P. Krantz, G. O. Samach, F. Yan, J. L. Yoder, K. Watanabe, T. Taniguchi, T. P. Orlando, S. Gustavsson, P. Jarillo-Herrero, and W. D. Oliver, Nat. Nanotechnol. 14, 120 (2019).

[19] H. Zhang, C.-X. Liu, X.-L. Qi, X. Dai, Z. Fang, and S.-C. Zhang, Nat. Phys. 5, 438 (2009).

[20] C.-X. Liu, X.-L. Qi, H. J. Zhang, X. Dai, Z. Fang, and S.-C. Zhang, Phys. Rev. B 82, 045122 (2010).

[21] D. Kong, J. C. Randel, H. Peng, J. J. Cha, S. Meister, K. Lai, Y. Chen, Z.-X. Shen, H. C. Manoharan, and Y. Cui, Nano Lett. 10, 329 (2010).

[22] S. S. Hong, W. Kundhikanjana, J. J. Cha, K. Lai, D. Kong, S. Meister, M. A. Kelly, Z.-X. Shen, and Y. Cui, Nano Lett. 10, 3118 (2010).

[23] R. Egger, A. Zazunov, and A. L. Yeyati, Phys. Rev. Lett. 105, 136403 (2010).

[24] A. Cook and M. Franz, Phys. Rev. B 84, 201105(R) (2011).

[25] A. M. Cook, M. M. Vazifeh, and M. Franz, Phys. Rev. B 86, 155431 (2012).

[26] Y.-J. Doh, J. A. van Dam, A. L. Roest, E. P. A. M. Bakkers, L. P. Kouwenhoven, and S. De Franceschi, Science 309, 272 (2005).

[27] R. M. Lutchyn, J. D. Sau, and S. Das Sarma, Phys. Rev. Lett. 105, 077001 (2010).

[28] Y. Oreg, G. Refael, and F. von Oppen, Phys. Rev. Lett. 105, 177002 (2010).

[29] J. Alicea, Y. Oreg, G. Refael, F. von Oppen, and M. P. A. Fisher, Nat. Phys. 7, 412 (2011).

[30] W. Chang, S. M. Albrecht, T. S. Jespersen, F. Kuemmeth, P. Krogstrup, J. Nygård, and C. M. Marcus, Nat. Nanotechnol. 10, 232 (2015).

[31] L. Fu and C. L. Kane, Phys. Rev. Lett. 100, 096407 (2008).

[32] I. Sochnikov, A. J. Bestwick, J. R. Williams, T. M. Lippman, I. R. Fisher, D. Goldhaber-Gordon, J. R. Kirtley, and K. A. Moler, Nano Lett. 13, 3086 (2013).

[33] S. Cho, B. Dellabetta, A. Yang, J. Schneeloch, Z. Xu, T. Valla, G. Gu, M. J. Gilbert, and N. Mason, Nat. Commun. 4, 1689 (2013).

[34] L. Galletti, S. Charpentier, M. Iavarone, P. Lucignano, D. Massarotti, R. Arpaia, Y. Suzuki, K. Kadowaki, T. Bauch,
A. Tagliacozzo, F. Tafuri, and F. Lombardi, Phys. Rev. B 89, 134512 (2014).

[35] M. Hell, J. Danon, K. Flensberg, and M. Leijnse, Phys. Rev. B 94, 035424 (2016).

[36] M. C. Dartiailh, T. Kontos, B. Douçot, and A. Cottet, Phys. Rev. Lett. 118, 126803 (2017).

[37] J. Manousakis, A. Altland, D. Bagrets, R. Egger, and Y. Ando, Phys. Rev. B 95, 165424 (2017).

[38] D. Pikulin, K. Flensberg, L. I. Glazman, M. Houzet, and R. M. Lutchyn, Phys. Rev. Lett. 122, 016801 (2019).

[39] X. Du, I. Skachko, and E. Y. Andrei, Phys. Rev. B 77, 184507 (2008).

[40] Y. Tanaka, T. Yokoyama, and N. Nagaosa, Phys. Rev. Lett. 103, 107002 (2009).

[41] J.-D. Pillet, C. H. L. Quay, P. Morfin, C. Bena, A. L. Yeyati, and P. Joyez, Nat. Phys. 6, 965 (2010).

[42] J. R. Williams, A. J. Bestwick, P. Gallagher, S. S. Hong, Y. Cui, A. S. Bleich, J. G. Analytis, I. R. Fisher, and D. GoldhaberGordon, Phys. Rev. Lett. 109, 056803 (2012).

[43] M. Snelder, M. Veldhorst, A. A. Golubov, and A. Brinkman, Phys. Rev. B 87, 104507 (2013).

[44] A. C. Potter and L. Fu, Phys. Rev. B 88, 121109(R) (2013).

[45] R. Ilan, J. H. Bardarson, H.-S. Sim, and J. E. Moore, New J. Phys. 16, 053007 (2014).

[46] D. J. van Woerkom, A. Proutski, B. van Heck, D. Bouman, J. I. Väyrynen, L. I. Glazman, P. Krogstrup, J. Nygård, L. P. Kouwenhoven, and A. Geresdi, Nat. Phys. 13, 876 (2017).

[47] A. Kringhøj, L. Casparis, M. Hell, T. W. Larsen, F. Kuemmeth, M. Leijnse, K. Flensberg, P. Krogstrup, J. Nygård, K. D. Petersson, and C. M. Marcus, Phys. Rev. B 97, 060508(R) (2018).

[48] F. Luthi, T. Stavenga, O. W. Enzing, A. Bruno, C. Dickel, N. K. Langford, M. A. Rol, T. S. Jespersen, J. Nygård, P. Krogstrup, and L. DiCarlo, Phys. Rev. Lett. 120, 100502 (2018).

[49] See Supplemental Material at http://link.aps.org/supplemental/ 10.1103/PhysRevB.100.241408 for additional details and derivations.

[50] A. Y. Kitaev, Phys. Usp. 44, 131 (2007).

[51] P. F. Bagwell, Phys. Rev. B 46, 12573 (1992).

[52] D. V. Averin, Phys. Rev. Lett. 82, 3685 (1999).

[53] J. M. Martinis, K. B. Cooper, R. McDermott, M. Steffen, M. Ansmann, K. D. Osborn, K. Cicak, S. Oh, D. P. Pappas, R. W. Simmonds, and C. C. Yu, Phys. Rev. Lett. 95, 210503 (2005).

[54] A. Wallraff, D. I. Schuster, A. Blais, L. Frunzio, R.-S. Huang, J. Majer, S. Kumar, S. M. Girvin, and R. J. Schoelkopf, Nature (London) 431, 162 (2004).

[55] D. I. Schuster, A. A. Houck, J. A. Schreier, A. Wallraff, J. M. Gambetta, A. Blais, L. Frunzio, J. Majer, B. Johnson, M. H. Devoret, S. M. Girvin, and R. J. Schoelkopf, Nature (London) 445, 515 (2007). 